\title{
Exploration of coping strategies among bereaved young adults using six-part story method (6PSM)
}

\author{
Merikan Aren ${ }^{1}$, Venessha Sambasivan ${ }^{1}$
}

${ }^{1}$ Faculty of Cognitive Sciences and Human Development, University Malaysia Serawak, Malaysia

\begin{tabular}{l} 
Article Info \\
\hline Article history: \\
Received Jan $15^{\text {th }}, 2021$ \\
Revised Feb $23^{\text {th }}, 2021$ \\
Accepted Mar $20^{\text {th }}, 2021$ \\
\hline
\end{tabular}

\section{Keyword:}

Bereaved young adult Coping strategies Six-part story method (6PSM)

\begin{abstract}
The purpose of this research was to explore the coping strategies among bereaved young adults using Six-Part Story Method (6PSM). More specifically, this study was meant to identify the strengths of coping using 6PSM and the suitability of 6PSM among young adults in counselling approach. Counselling session was adapted as a medium for data collection with the aid of 6PSM as an intervention technique. Purposive sampling and snowball sampling was used to identify the most suitable participants to contribute in this study. The ethics of research and counselling such as anonymity, confidentiality, information disclosure, and the acquisition of informed consent were followed strictly throughout this research. The data collected was then analyzed using content analysis by categorizing the data into themes that present in the BASICPh model introduced by Mooli Lahad (Belief, Affect, Social, Imagination, Cognitive, and Physical) Other than that, this study had also showed that themes such as belief, affect, and social are highly used among young adults as a way of dealing with bereavement. Not only that, this study had also found that the process of completing 6PSM enables an individual to express themselves in a way that synchronizes with the BASICPh model of coping. Based on the findings, this study would like to implicate that the coping strategies of bereaved young adults falls under the themes of belief, affect, and social. In addition, it also implicated that 6PSM is suitable to be used as an intervention in counselling young adults.
\end{abstract}

(C) 2021 The Authors. Published by IICET.

This is an open access article under the CC BY-NC-SA license (https://creativecommons.org/licenses/by-nc-sa/4.0

\section{Corresponding Author:}

Aren, M.,

Faculty of Cognitive Sciences and Human Development, University Malaysia Serawak, Malaysia

Email: amerikan@unimas.my

\section{Introduction}

Death of someone loved is a life process most adults will go through at least once in their lifetime. The deceased could be their friend, family, relative or even colleague. According to the Department of Statistics (2018), approximately 168168 deaths in Malaysia were recorded in the year 2017. The crude death rate was said to be increased as much as 0.2 per 1000 population in which it went from 5.1 (2016) to 5.2 (2017) (Department of Statistics, 2018).

In conjunction to that, an interpersonal emotional response known as grief is developed (Staar, 2018). As an expression of grief, mourning that is geographically and culturally influenced takes place (Thai \& Moore, 2018). According to Bogan (2019), the combination of both grief and active mourning results in bereavement in which an individual may experience psychological, physiologic, or behavioural changes in a long run. 
When an individual undergoes bereavement, coping strategies plays a major role in helping them to overcome that heart wrecking situation. Coping strategies in this context is defined as a set of focused and volunteered effort that normalizes an individual's internal and external aspects when they are dealing with overwhelming emotions (Kulari, 2017). However, coping strategy does not work on its own. According to Staar (2018), the process of working through grief is not only determined by an individual's effort alone but it's determined by the support system of that individual as well. The way a person grieves is affected by how his or her expression of emotions and thoughts are being perceived and that is determined through the quality of communication (Staar, 2018).

Therefore, some counsellors recommend the use of art therapy when it comes to counselling a bereaved individual. This is because, art therapy is an attractive intervention which enables and encourages a particular individual to express their thoughts and feelings that are out of their awareness and verbal abilities (Dye, 2018). Kulari's (2017) study which was focused on art therapy techniques to improve coping strategy in children has proven that through the use of art intervention, children are able to describe their overwhelming emotions more openly and manifestation of strong coping strategy has taken place in them as well. In addition, Staar's (2018) research also has implicated that art therapy provides an individual the chance to discover and voice out their overwhelming emotions without any expectation or fear of rejection from others through art and the art making process.

Taking into consideration of the struggle faced by bereaved individuals and statistical data of crude death rate, adults specifically young adults are at risk when it comes to the loss of a loved one (Pitman, Rantell, Marston, King, \& Osborn., 2017). Therefore, this study focuses on bereaved young adults because they are undergoing a critical period of life transition. According to Erikson's Theory, young adults belong to the "Intimacy vs. Isolation" stage in which intimate expression of one's self takes place (McLeod, 2018). Thus, if there's a crisis of loss, that individual may undergo maladaptive development especially when their support system is inadequate to assist with coping. Hence, this research is carried out to explore the coping strategies among bereaved young adults through the use of Six-Part Story Method (6PSM) intervention under the art therapy approach

Being deprived from the loss of a close relation to death is generally universal. The internal challenges and struggles encountered by individuals who have lost their loved ones has been well-documented in recent years. According to Pitman et al. (2017), sudden bereavement often causes an individual to be stigmatised by the loss. This experience of stigma, embarrassment, and masking the cause often puts an individual at risk of committing suicide especially when the death is traumatizing (Pitman et al., 2017). The development of suicidal thoughts takes place due to the perceived stigma from other's rudeness and also as a response from the socioemotional and cognitive impact in conjunction to the loss (Pitman et al., 2017). The situation mentioned above can be experienced by all individuals be it children, adolescents, young adults, adults or even elderlies. However, by enhancing the support system (ex: counselling) for the bereaved individuals, the possibilities of suicidal attempts could be reduced (Pitman et al., 2017).

The loss of close relation is a universal equalizer in which most individuals will undergo bereavement. Bereavement also acts as a cause of psychological distress in an individual (Thai \& Moore, 2018). According to Balk as cited in Thai and Moore (2018), the psychological distress faced by an individual is said to be linear to the bonding with the deceased. In simpler words, the closer the individual is with the deceased, the more the psychological distress experienced by him or her. This overwhelming emotions combines with the lack of understanding or knowledge in adapting to the loss resulting in difficulties to develop coping strategies (Thai \& Moore, 2018). Failure to develop those coping strategies will eventually expose an individual to the possibilities of being physically, socially, cognitively and emotionally disturbed (Thai \& Moore, 2018). In conjunction to that, an individual, students specifically are at a higher risk of performing badly in academics or even being a school dropout. However, the occurrence of this situation can be reduced if there is proper exposure to guidance and insights to develop coping strategies. With that being said and taking into consideration of the importance of coping strategies, this study's objectives are deliberated as to explore the coping strategies among bereaved young adults, to identify the strengths of coping using Six-Part Story Method (6PSM) and to identify the suitability of Six-Part Story Method (6PSM) among young adults in counselling approach.

\section{Method}

The research design used in this study was a qualitative research design. Qualitative research design is an interpretive and naturalistic approach in the research field (Aspers \& Corte, 2019). Undergraduate students of University Malaysia Sarawak (UNIMAS) were selected to be the population for this study. The setting, 
population and sample was chosen as above because students from UNIMAS represent almost all the states in Malaysia as they are originated from the east and west Malaysia. In addition, most of them fall under the young adult category. Hence, the representation of young adults from different parts of Malaysia is ensured and the way they grief, mourn and cope differ from one another as it is influenced by both geographical and cultural factors (Staar, 2018). Therefore, this was an advantage to the researcher as it provided a platform to identify if the six part story method (6PSM) intervention under the art therapy approach is suitable to be used on bereaved young adults in general.A sum of 8 respondents from the population were chosen using purposive sampling and snowball sampling. Purposive sampling is a non-random sampling technique used to select specific information-rich participants that possesses qualities or fulfil the criteria required for a particular study (Ilker Etikan, Sulaiman Abubakar Musa, \& Rukayya Sunusi Alkassim, 2016). This technique was adapted in this study because only information-rich participants who could fulfil all the criteria mentioned earlier can become the sample for this research. As for snowball sampling, Edmonds (2019) stated that it is a sampling technique that generates a linkage of eligible participants through the suggestion of readily selected samples. This technique was significant for this study because the help of the readily selected participants were required to identify additional samples who were eligible.

There were some criteria that the participants need to meet to be eligible for this study. First of all, the respondents were required to have lost at least one loved individual to death. That individual can be an immediate family member, a friend, colleague, or anyone whose absence had affected the participant. Secondly, the death period of that deceased individual should not exceed or must be within the time span of 3 years. The time span was allocated as stated because within that period, an individual will still be going through acute grief (Thai \& Moore, 2018). Phase of bereavement called acute grief is an initial reaction to the loss of someone close in which it is often intense and unsettling (Shear, Ghesquiere \& Glickman, 2013). Therefore, this period of bereavement will ensure that the individual's intensity in bereaving is reliable or credible for this study. Last but not least, the participants were also required to be young adults in which they must be within the age group of 18 to 35 years old (Barrington et.al., 2018). One of the instrument that was used in this study was semi-structured questions. All the participants were asked a set of similar semistructured questions regarding their background and the deceased, and the process of bereavement experienced. The semi-structured questions were mostly open-ended questions and the participants were allowed to share their thoughts and feelings openly for a more lenient process of sharing.

Since the focus of this research was to explore the coping strategy of the bereaved young adults, a group counselling session was carried out using art therapy approach with the aid of Six-Part Story Method (6PSM) as an intervention technique. In conjunction to that, Lahad as cited in Kirk (2017) developed an integrative model of coping and resiliency, BASICPh (Belief, Affection, Social, Imagination, Cognitive, and Physical) to analyse the coping strategies. This was the third instrument used in this study in which it was used as guide to assess those traumatised and stressed individuals' coping strategies by classifying the key words mentioned by the participants in their story into the themes presented in this model (Jennings, 2012). Through that, the strongest theme is identified and that marks as the individual's coping strategy (Jennings, 2012). Since both 6PSM and BASICPh model are interrelated and suitable to be used as an instrument in treating stressed and traumatised human beings, this study had also used 6PSM and BASICPh in conducting the group counselling session as well as analysing the coping strategies of the individuals.

\section{Results and Discussions}

The findings of this research was coded into six themes present in the BASICPh model such as belief, affect, social, imagination, cognition, and physical. The coded data is presented in a way that aligns with the objectives of the study.

What are the coping strategies among bereaved young adults?

Table 1. Coping strategies of bereaved young adults

\begin{tabular}{|c|c|c|c|c|c|c|}
\hline $\begin{array}{c}\text { Respondent } \\
\text { A }\end{array}$ & Belief & Affect & $\begin{array}{r}\text { Social } \\
X\end{array}$ & $\underset{X}{\text { Imagination }}$ & $\begin{array}{c}\text { Cognition } \\
X\end{array}$ & Physical \\
\hline B & $\mathrm{X}$ & & $X$ & & & X \\
\hline C & & X & X & & $\mathrm{X}$ & \\
\hline D & $\mathrm{X}$ & & X & & & $\mathrm{X}$ \\
\hline E & $\mathrm{X}$ & $\mathrm{X}$ & & & & $X$ \\
\hline F & $\mathrm{X}$ & & & & & $\mathrm{X}$ \\
\hline G & & X & X & & & X \\
\hline $\mathbf{H}$ & $\mathrm{X}$ & $X$ & $\mathrm{X}$ & & & \\
\hline
\end{tabular}


Table1. above displays the coping strategies of the respondents based on the themes present in the $\mathrm{BASICPh}$ model. Based on the table, respondent A was found to exhibit social, imagination and cognition coping strategies through her storyline and it was presented as below. As for respondent $\mathrm{B}$ and $\mathrm{D}$, they had expressed belief, social and physical as their coping strategies. Next, respondent $\mathrm{C}$ was found to have expressed affect, social, and cognition as her coping strategies. On the other hand, the coping strategies expressed by respondent $\mathrm{E}$ was belief, affect, and physical. Respondent $\mathrm{F}$ had only exhibited two coping strategies which is belief and physical. As for respondent $\mathrm{G}$, she had expressed affect, social and physical as her coping strategies. Lastly, respondent H's coping strategies were found to be belief, affect, and social.

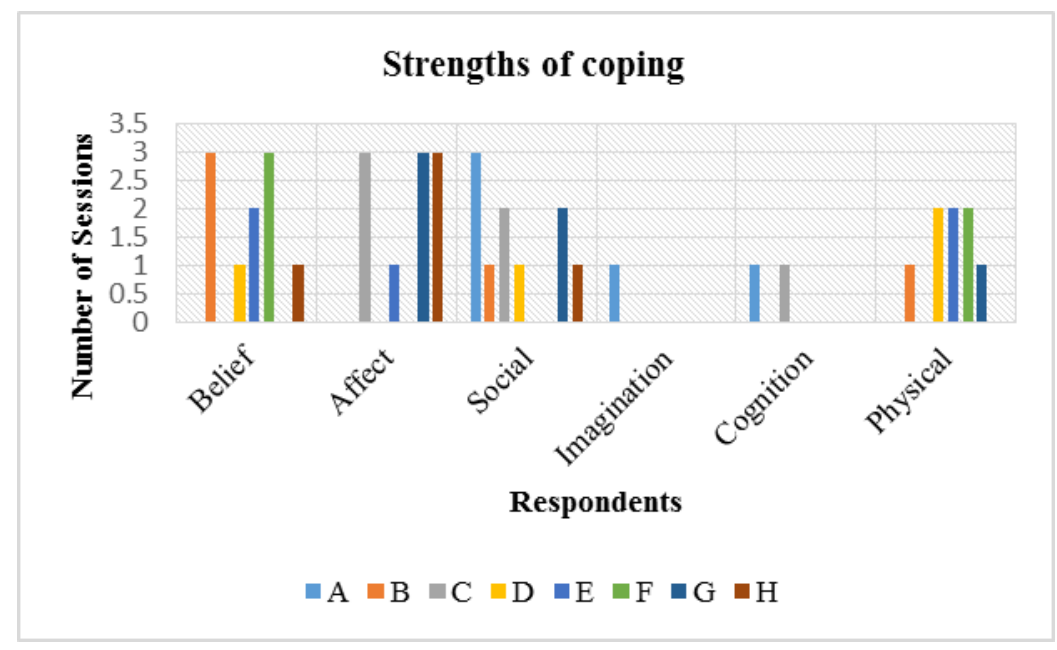

Figure 1. The strengths of coping

Figure 1. Shows the strengths of coping of the respondents based on BASICPh themes. Based on the figure, respondent $B$ and $F$ have portrayed high belief, meanwhile respondent $C, G$ and $H$ were found to have high affect from all the sessions. As for social, only respondent A had recorded it high throughout the sessions. As for imagination, also only respondent $\mathrm{A}$ had portrayed it but it was found to be low. Moving on, only respondent $\mathrm{A}$ and $\mathrm{C}$ had portrayed cognition and it was found to be low as well. Lastly, respondent $\mathrm{D}, \mathrm{E}$ and $\mathrm{F}$ had marked a medium level of physical as their coping strategy throughout the sessions.

\section{Is Six-Part Story Method (6PSM) suitable to be used among young adults in counselling approach?}

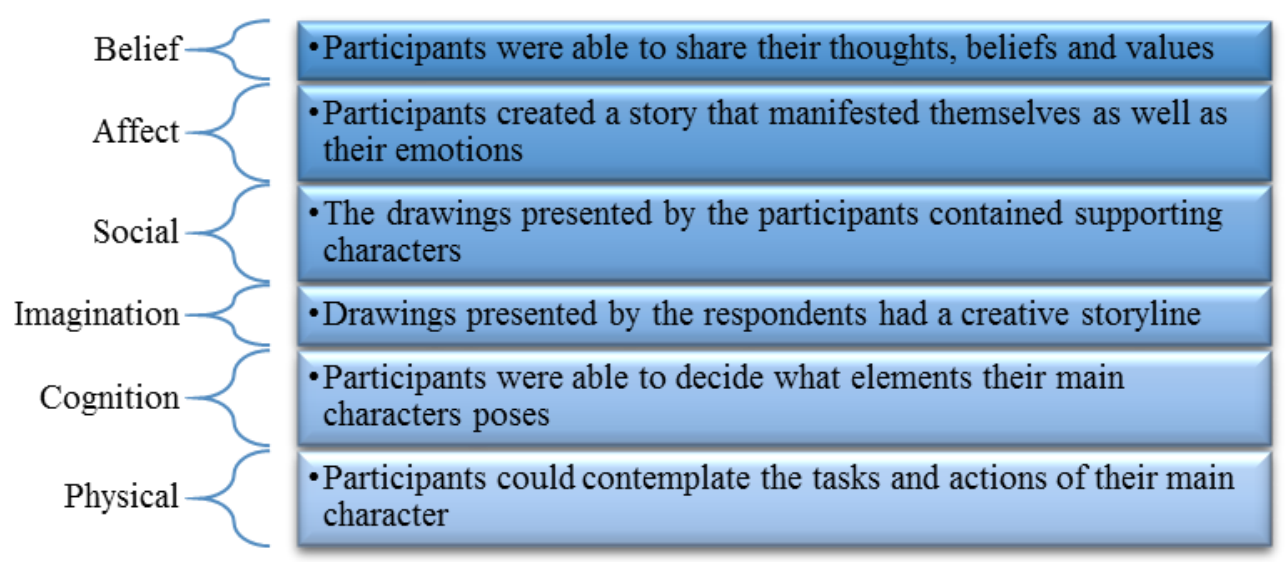

Figure 2. Observation based on the respondents' storyline

Figure 2. deliberates the researcher's observation on the storyline of the respondents. Based on the observation, it was found that the respondents' storyline and drawing had complied with the BASICPh model of coping. For example, the respondents had expressed belief in which they were able to portray their thoughts, beliefs, and values in their story. They had also expressed affect by creating a story that manifested themselves as well as their emotions. Not only that, the appearance of supporting characters in their story and the creation of a creative storyline had reflected the use of social and imagination respectively. Other than that, the theme cognition can also be observed when the respondents were able to assign tasks and elements for 
their characters accordingly. Last but not least, the respondents' ability to contemplate the tasks and actions of the characters had portrayed the use of physical as a coping strategy.

\section{DISCUSSION}

Objective 1: To explore the coping strategies among bereaved young adults

\begin{tabular}{|c|c|}
\hline Respondent A & -Affect, Imagination, Cognition \\
\hline Respondent B & -Belief, Social, Physical \\
\hline Respondent C & -Affect, Social, Cognition \\
\hline Respondent D & •Belief, Social, Physical \\
\hline Respondent $\mathrm{E}$ & -Belief, Affect, Physical \\
\hline Respondent F & •Belief, Physical \\
\hline Respondent G & -Affect, Social, Physical \\
\hline Respondent $\mathrm{H}$ & -Belief, Affect, Social \\
\hline
\end{tabular}

Figure 3. Coping Strategies of the respondents

Based on the findings, the coping strategies of the bereaved individuals who participated in this study were categorised into themes as presented in figure 4.5. According to the figure, it can be concluded that most of the participants overcome their grief using utmost three types of coping strategy.

A study by Lahad, Shacham and Ayalon (2012) had stated that an extrovert individual who is responsible and open to experiencing new things usually depicts coping strategies that falls under affect, imagination and cognition. This statement complies with respondent A's coping strategies which is affect, imagination, and cognition as well because her storyline was mostly about the people around her and they had goals and responsibilities of their own. Next, individuals who cope through belief, social and physical are those who are goal orientated and said to be merely focused on their actions (Lahad et al., 2012). This aligns with respondent $\mathrm{B}$ and $\mathrm{D}$ because they did mentioned about the things they need to do in order to achieve their goal. Thus it can be concluded that their storyline does comply with their coping strategies which is belief, social and physical.

On the other hand, Lahad et al (2012) also indicated that an individual who copes through affect, social and cognition have high emotional well-being because they are capable of expressing their feelings openly. This actually synchronises with respondent C's storyline because her story was focused on family, feelings, and the way to express those feelings. Therefore, respondent $C$ is implicated to cope through affect, social, and cognition. According to Klohnen as cited in Lahad et al. (2012), productiveness, optimism and skilled expressiveness was believed to indicate coping strategies that falls under belief, affect, and physical. Therefore, respondent $\mathrm{E}$ was placed under those themes owing to the fact that she had expressed the ways to overcome her problems throughout her story.

Furthermore, Lahad et al. (2012) had also stated that an individual who expresses the means of coping multiple risk factors or stressors will usually fall under the theme belief and physical. Respondent $\mathrm{F}$ had complied with this statement because his storyline was mainly focused on the things that he need to do in order achieve his goal and the possible barriers that could stop him from achieving that goal were also listed accordingly. Thus, respondent $\mathrm{F}$ was indicated to cope through belief and physical coping strategies.

According to Martinez-Torteya as cited in Lahad et al. (2012), an uncomplicated family relationship despite the presence of discorded parents and the portrayal of de-escalation or self-control is indicated to fall under affect, social and physical coping strategies. This actually synchronises with respondent G's storyline that mainly expressed the importance of self-control in dealing with the conflicts at home and thus her coping strategies were aligned so. 
Last but not least, the expression of anticipation, altruism, self-observation, and sublimation were classified under the themes belief, affect, and social by Skodol as cited in Lahad (2012). Respondent H's storyline contained almost all of these concepts in which the story revolved around the main character and how she sees herself and the people surrounding in a positive manner despite their negative opinions and comments. Thus, respondent $\mathrm{H}$ was classified to portray belief, affect, and social as her coping strategies.

\section{Objective 2: To identify the strengths of coping using Six-Part Story Method (6PSM) Belief}

Table 2. Strengths of belief

\begin{tabular}{lc}
\hline Strength & Respondents \\
High & B and F \\
Medium & E \\
Low & D and H \\
\hline
\end{tabular}

Blief is an aspect of resilience that brings clarity to a society, group, or an individual (Cohen, 2003). The representation of one's self, accompanied by overflowing ideas are often associated as way of coping through belief (Lahad, 2017). In conjunction to that, Lahad (2017) had stated that consistent clarity, value, and meaning conveyed by an individual reflects a high level of belief and it is often linked with Abraham Maslow's hierarchy of needs. Based on the table 4.3 above, respondent $\mathrm{B}$ and $\mathrm{F}$ had presented high level of belief because all three of their stories represented themselves accompanied by overflowing ideas that contained clarity, meaning, and values. Meanwhile, respondent E's storyline had high self-ideology, value, and meaning but there was a lack clarity and thus she expressed a medium level of belief. As for respondent $\mathrm{D}$ and $\mathrm{H}$, they had recorded low level of belief because their story had value and meaning but the concept of self-ideology and clarification was lacking.

\section{Affect}

Table 3. Strengths of affect

\begin{tabular}{lc}
\hline Strength & Respondents \\
High & $\mathrm{C}, \mathrm{G}$ and $\mathrm{H}$ \\
Low & $\mathrm{E}$
\end{tabular}

Sigmund Freud had spoken about the affectionate world in one of his writings in which it was stated that emotional well-being determines the way an individual perceives the world. This transacts an individual's reality because they tend to manifest their emotions as a strategy to get through life as well as to cope grief (Lahad 2017). By validating those feelings and emotions, an individual will be able to share their fear, sorrow and anger (Cohen, 2003). This can be seen in respondent C, G, and H as they were recorded to have conveyed a high level of affect referring to table 4.4. This is due to the fact that they had expressed high level of feelings, ventilations, acceptance, and emotional well-being while narrating all three of their stories. On the other hand, respondent $\mathrm{E}$ recorded to have conveyed low level of affect because she had only expressed feelings and acceptance but not ventilations or emotional wellbeing.

Social

Table 4. Strengths of social

\begin{tabular}{ll}
\hline Strength & Respondents \\
High & A \\
Medium & C and G \\
Low & B, D and H \\
\hline
\end{tabular}

According to Cohen (2003), social aspect was described as a sense of belonging to a part of a group and having sense of responsibility within that group. A storyline that focuses on social role systems, social skills, and assertiveness as highlighted by Erik Erikson under the role of society in his eight stages of development was said to reflect a high level of affect (Lahad, 2017). Based on the table 4.5, only respondent A had expressed high level of social for the reason that all of her drawings were only focused on a third person but not herself. Not only that, the stories had also elaborated the concept of social role systems, social skills, and assertiveness very well. Meanwhile, respondent $\mathrm{C}$ and $\mathrm{G}$ had expressed a medium level of social because their stories was not. 
Cognition

Table 5. Strength of cognition

\begin{tabular}{ll}
\hline Strength & Respondents \\
\hline Low & A \\
\hline
\end{tabular}

Cognitive element basically utilises knowledges, facts and strategies when it comes to problem solving or coping (Cohen, 2003). Lazarus's cognitive-mediational theory had illustrated that the rendition of problem solving skills, reality, and knowledge is perceived to be a reflection of cognition as a coping strategy (Lahad, 2017). Referring to table 4.7, respondent $A$ and $C$ were recorded to have a low level of cognition owing to the fact that there was a lack of consistency in the expression of problem solving skills, reality and knowledge as it was only presented in one of their story

\section{Physical}

Tabel 6. Strength of physical

\begin{tabular}{ll}
\hline Strength & Respondents \\
Medium & D, E, and F \\
Low & B and G \\
\hline
\end{tabular}

The word physical alone refers to the actions of doing something to keep one's self occupied (Cohen, 2003). Pavlov's and Watson's findings on behaviourism had explained that the expression of actions, work, or activities often indicates the use of physical as a coping strategy (Lahad, 2017). Based on table 4.8, respondent $\mathrm{D}, \mathrm{E}$, and $\mathrm{F}$ were recorded to be at the medium level for the reason that they have conveyed all the elements mentioned above accordingly in most of their story. However, not all their stories contained a consistent expression of the physical as coping strategy and so it was classified to be moderate. Meanwhile, respondent $B$ and $\mathrm{G}$ were recorded to have a low level of physical because they had only mentioned the actions or work in one or two of their stories but did not express it accordingly.

\section{Objective 3: To identify the suitability of Six-Part Story Method (6PSM) among bereaved young adults in counselling approach}

The use 6PSM had encouraged each client to express themselves through their drawing and story. A research by Stephenie (2020) stated that this intervention opens up a space for individuals to share what they consider important throughout their life. It is agreeable because based on the researcher's view, the respondents were able to share their beliefs and values by engaging in this activity that allowed them to create their own drawing and story. Besides that, Stephanie (2020) had also emphasized that this play allows the respondents to represent the feelings of their characters and guides. That is undeniable for the reason that the respondents indeed were able to create a story that manifested themselves or things around them as well as their emotions. Not only that, Stephanie (2020) had also deliberated that 6PSM allows the expression of social support concept whenever the respondents explore the supporting characters for their story. This can be applicable for this study as well because the drawings presented by the respondents did have some supporting characters who held on to their roles accordingly.

In addition, the study by Stephanie (2020) had also illustrated the involvement of imagination in this 6PSM activity owing to the fact that the respondents will be required to imagine the storyline of their drawing. This statement also synchronises with this study because the respondents created a creative storyline to represent their drawing. Furthermore, Stephanie (2020) had also emphasized that 6PSM encourages the use of cognition as the respondents decide what elements their main characters should poses. The use of physical elements in this activity were also deliberated by Stephanie (2020) by referring to the fact that the individuals will be required to personify the characters and guides through their storytelling. These two statements does comply with this research as well because the use of cognition and physical elements can also be observed from the respondent's storyline as they contemplated the tasks and actions of the characters in their story very well. As a whole, it can be observed that 6PSM is applicable in counselling approach because it allows the clients to 
express themselves in a way that synchronises with the BASICPh model of coping that may eventually help in the analysis of an individual's coping strategy. Thus, it can be concluded that Six-Part Story Method (6PSM) is suitable to be used in counselling young adults

\section{Conclusion}

Based on the findings of the research, coping strategies such as belief, affect and social were often used by young adults and it was recorded to be at a high level. Physical was used moderately, meanwhile cognition and imagination were used the least among young adults to cope their grief. Although all the coping strategies represented by the BASICPh model was used by the individuals at some point, this study still implicates that the coping strategies of bereaved young adults falls under belief, affect, and social because it was used vastly compared to the other coping strategies. Not only that, these three strategies were also conveyed consistently throughout all the stories of most of the respondents. Furthermore, the study had also concluded that Six-Part Story Method (6PSM) is suitable to be used as an intervention among young adults in counselling approach. It was derived so for the reason that the young adults who participated in this research were able express themselves through their drawings and their storylines. They were able to portray most of the coping strategies listed in BASICPh model throughout the process of this activity as well as their drawing and story. As mentioned by Stephanie (2020), this activity had allowed those individuals to open up themselves and share what they consider important throughout their life process. Although this statement was directed to children, it was clearly applicable to young adults in this context. Thus, this study had implicated that 6PSM is suitable to be used as an intervention among young adults in counselling approach.

\section{References}

Aspers, P., \& Corte, U. (2019). What is qualitative in qualitative research. Qualitative Sociology. 42, 139-160

Barrigton, G., Bettiol, S., Barnett, T., Crocombe, L., \& Khan, S. (2018). Is overweight/obesity a risk factor for periodontitis in young adults and adolescents?: a systematic review. Obesity Reviews. 19(6), 852-883

Bhar, S. (2019). nological research methodology in sustainable consumption literature:Illustrations from India. International Journal of Qualitative Methods. 18, 1-14

Bogan, B. (2019). Exploring the usage of found objects in art therapy for bereavement: A literature review. Expressive Therapies Capstone Theses. 209, 1-34

Brooten, D., Youngbelt, J. M., Caicedo, C., Moral, T. D., Cantwell, G. P., \& Totapally, B. (2018). Parents' acute illnesses, hospitalizations, and medication changes during the difficult first year after infant or child NICU/PICU death. American Journal of Hospice \& Palliative Medicine. 35(1), 75-82

Cohen, A. (2003). Coping with stress and surviving challenging times. Child Care Information Exchange. Retrieved fromhttp://www.oas.org/udse/readytolearn/Israel.pdf

Darlington, A. E., Korones, D. N., \& Norton, S. A. (2018). Parental coping in the context of having a child who is facing death: A theoretical framework. Palliative and Supportive Care. 16, 432-441

Department of Statistics Malaysia. (2018). Statistics on causes of death, Malaysia, 2018. Retrieved from https://www.dosm.gov.my/v1/index.php?r=column/cthemeByCat\&cat=401\&bul_id=aWg2VjJkZHh DdEM3JQSGloeTV1Zz09\&menu_id=L0pheU43NWJwRWVSZklW zQ4TlhUUT09

Djelantik, M. J., Robinaugh, D. J., Kleber, R. J., Smid, G. E., \& Boelen, P. A. (2019). Symptomatology following loss and trauma: Latent class and network analyses of prolonged grief disorder,posttraumatic stress disorder, and depression in treatment-seeking trauma-exposed sample. Depress anxiety. 1-9

Dye, M. (2018). Evaluating the benefits of art therapy interventions with grieving children. Educational Specialist.129, 2-41

Edmonds W. M. (2019). Snowballing Prayforme: A qualitative study using snowball sampling. London, UK: SAGE Publications Ltd.

Griffiths, A., Coackley, A., Cooper, M., Watson, A., Benson, S., Cadwallader, C., ...Hampton-Matthews, J. (2019). Innovative four step bereavement service at a tertiary cancer centre. BMJ Support Palliat Care. 9(26), 1-71

Ilker Etikan, Sulaiman Abubakar Musa, \& Rukayya Sunusi Alkassim. (2016). Comparison of convenience sampling and purposive sampling. American Journal of Theoretical and Applied Statistics. 5(1). 1-4

Jennings, S. (2012). Neuro-dramatic-play and trauma 'towards healing and hope'. Penang, MY: B. BraunMedical Industries

Kirk, K. (2017). Storymaking, psychodrama, and happy ever after: Using children's stories to create dramas and find other endings. Z Psychodrama Soziom. 16, 95-106 
Kulari, G. (2017). Art therapy techniques to improve coping strategies in children 7-18 years old with a chronic disease. (Unpublished doctorate's thesis). Universidade Nova de Lisboa, Lisbon, Portugal.

Lahad, M. (2017). From victim to victor: The development of the BASIC PH model of coping and resiliency.Traumatology, 23(1), 27-34

Lahad, M., Shacham, M., \& Ayalon, O. (2012). The "BASIC Ph" model of coping and resiliency. Retrievedfromhttps://books.google.com.my/books?id=TW_7bzdC3oC\&printsec=frontcover\&dq=Basi $\mathrm{cph}+$ modl+for+grief\&hl=en\&sa=X\&ved=2ahUKEwiQ5cPqbqAhVT8XMBHYAnAc4Q6AEwAHoE CAIQAg $\mathrm{v}=$ onepage $\& \mathrm{q} \& \mathrm{f}=$ false

McLeod, S. A. (2018). Erik Erikson's stages of psychosocial development. Retrievedfromhttps://www.simplypsychology.org/Erik-Erikson.html

Pitman, A., Rantell, K., Marston, L., King, M., \& David, O. (2017). Perceived stigma of sudden bereavement as a risk factor for suicidal thoughts and suicide attempt: Analysis of British cross-sectional survey dataon 3387 young bereaved adults. International Journal of Environment Research and Public Health. 14(286), 1-12

Popescu, M. (2018). Elements aiming to optimize coping strategies in pupils by the use of interventionalprogrammes. Journal of Experiential Psychotherapy. 21(82), 29-39

Shear, M. K., Ghesquiere, A., \& Glickman, K. (2013). Bereavement and complicated grief. Curr PsychiatryRep, 15(11), 1-13

Staar, J. O. (2018). Art therapy with grieving adolescents in an impatient setting: An exploration of media andmaterials. (Published master's thesis). Drexel University, Philadelphia, Pennsylvania.

Stephenie, L. (2020). Re: Traumatizing - the interactions between clinicians and therapeutic helpers andvicarious trauma. Retrieved from Expressive Therapies Capstone Theses. (AAT 342)

Thai, C. L., \& Moore, J. F. (2018). Grief and bereavement in young adult college students: A review of the literature and implications for practice and research. Communication Research Trends. 37(4), 4-29. 